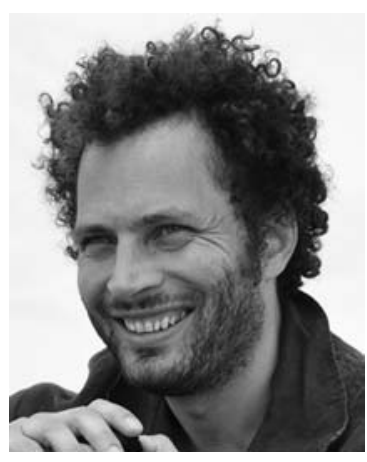

\title{
A Conversation with Julius Brennecke
}

\author{
Interviewer: RHIANNON MACRAe \\ Editor, Trends in Genetics
}

Julius Brennecke is a Senior Investigator at the Institute of Molecular Biotechnology of the Austrian Academy of Sciences.

Rhiannon Macrae: Could you introduce us to some of the work that you did here at Cold Spring Harbor?

Dr. Brennecke: At the time I joined Greg Hannon's group at CSHL, small RNA biology - also known as RNA interference-was a research topic on the rise. Greg Hannon was one of the big figures in this field, and he started to investigate a germ cell-specific class of small RNAs called PIWI-interacting RNAs (piRNAs). Those turned out to be the master control guardians to repress transposable elements, or selfish genes, in animals.

Rhiannon Macrae: Can you explain this idea of transposons as selfish genetic elements and where they come from and what they are doing?

Dr. Brennecke: Essentially every eukaryotic genome contains a variable amount of selfish genes, or transposable elements. These are stretches of DNA that carry genetic information that allows them to produce proteins that copy themselves and insert the copies into another location in the genome, which can be harmful to the cell or organism. It's probably one of the oldest, if not the oldest, genetic conflict on Earth. Ever since these transposons entered genomes, it has been a problem for host organisms to repress their activity. One way to repress the activity of transposable elements is using small RNAs.

Rhiannon Macrae: How widespread are piRNAs? Are they in all animals, in all eukaryotes?

Dr. Brennecke: Small RNAs are not animal-specific. Plants, fungi, animals - all use small RNA pathways to suppress transposable elements, but there are very different types. I think, more or less, every animal will have piRNAs. Different species have slightly different nuances, and sometimes you find very different features and patterns. Because biological research focuses so much on model organisms, we are only beginning to understand not just the similarities, but also the differences across species. We already know from the few animal systems we study that there is a huge diversity. The nematode
Caenorhabditis elegans has piRNAs, but they function in a completely different manner.

Rhiannon Macrae: Does C. elegans use some other small RNA to repress transposons?

Dr. Brennecke: They're still called piRNAs, because by definition they are PIWI-interacting and the proteins that bind to them in C. elegans belong to the PIWI clade of proteins. But we haven't identified a single protein that is shared between the worm pathway and the other studied animal piRNA pathways.

Rhiannon Macrae: Does that suggest convergent evolution of small RNA pathways? All eukaryotes have transposable elements, they all have to develop some kind of guardian against them, but it seems like different organisms have taken different routes to get there.

Dr. Brennecke: There is one common principle that is found in every small RNA pathway: You always have a small RNA that is bound to an Argonaute protein, and that guides this complex in a sequence-specific manner to a target RNA so that it can be silenced. This is also true for the piRNA pathway. But in the germline, for whatever reason, evolution juggled with this general principle and adopted different systems, different proteins, different ways to make the small RNA, onto the basic pathway and also determined whether the Argonaute protein acts in the nucleus or the cytoplasm. It's one of the most fastevolving systems that I know of, maybe as a consequence of this fight between transposons and genomes.

Rhiannon Macrae: Are transposable elements in the germline a particularly difficult problem to overcome, or because of the high risk do eukaryotic organisms have the most pressure to repress transposable elements in the germline?

Dr. Brennecke: By and large, the piRNA pathway is most active in the gonads. We don't have satisfying answers why transposon control is not as important in somatic cells. We know that transposable elements in 
mammals are silenced at the level of DNA methylation, so they are basically shut down to a large extent throughout the body, whereas in the germline where you have rounds of demethylation, it's a more vulnerable situation. Also, the germline genome is the most important one. Host organisms have a huge interest in protecting that genome. In evolutionary terms, there's limited value for a transposon to be able to transpose in somatic cells. In fact, it might even harm the host and thereby limit the evolutionary success of the transposon. It's clear that transposon promoters have evolved to be particularly active in germ cells. It's basically the battleground for their entire biology.

Rhiannon Macrae: Could you tell us about your more recent work in your own lab in Vienna?

Dr. Brennecke: In contrast to the much better understood microRNA pathway or short-interfering RNA pathway, where at most 10 proteins are specifically involved, the piRNA pathway has to deal with 40 to 50 proteins. There's definitely greater complexity. One of the focuses of my lab is how a cell actually manages to distinguish transposon RNA from any other cellular RNA. In terms of silencing, that's actually very easy because if a cell manages to put a small RNA into a PIWI family protein and that RNA is complementary to a transposon, the specificity problem is solved, but that just shifts the problem up one level: How do you make the small RNA? How does the cell distinguish the precursors that will end up in piRNAs versus other mRNAs? Our recent work suggests that the pathway couples the silencing step with the piRNA biogenesis step. If the cell has a transposon transcript and a piRNA is guiding a PIWI clade protein to a transcript that will lead to cleavage, the entire $3^{\prime}$ end of that transcript will make new piRNAs. Those piRNAs will be loaded into a nuclear PIWI family protein that will transport to the nucleus and exert its silencing function there.

Another area of our research is determining how the loci in the genome that encode piRNA precursors are transcribed, because it happens that these are within the heterochromatin, which typically is transcriptionally inert.

Rhiannon Macrae: Is that the case in the germline? If you're going through demethylation or reprogramming, are those still in a heterochromatic region, or at that point would that region be more accessible because the region is being transcribed and that's the point you would most need the piRNAs? Is there something special about germ cells that allow transcription of these heterochromatin loci?

Dr. Brennecke: Heterochromatin is defined by chromatin modifications. One of the most powerful modifications for transposon heterochromatin is histone $\mathrm{H} 3$ lysine 9 methylation. In all cells, this typically brings down a protein called HP1 that recognizes this chromatin mark and somehow recruits other factors to shut down the entire area for transcription or for the access of transcription factors. DNA methylation as a strong silencing epigenetic mark is not present in Drosophila, which is the model system my lab works on. Instead, in Drosophila there's an ingenious solution to the problem. Bill Theurkauf discovered an HP1-family protein in Drosophila germline cells called Rhino. It acts on the same heterochromatin loci by piggybacking a bunch of other germline-specific proteins that literally trick RNA polymerase II into fitting down in these areas and starting to randomly transcribe. We thought that such a highly specific process would be deeply conserved. However, the five proteins that are involved in this pathway are Drosophila-specific; we don't even find them in the honeybee.

Rhiannon Macrae: Do honeybees use DNA methylation? In other words, Drosophila might have this atypical pathway because it doesn't use DNA methylation, whereas other organisms that use DNA methylation as a silencing mechanism use a different system.

Dr. Brennecke: The connection that we currently see is that Drosophila has three PIWI family proteins that do the silencing job, and one of them is in the nucleus. This nuclear PIWI family protein is specific to Drosophila; other systems, like the silkworm Bombyx mori, for example, only have cytoplasmic PIWI family proteins. The nuclear PIWI family protein is important for Rhino's interaction with heterochromatin because PIWI instructs where Rhino should function. Without the nuclear PIWI family protein, the entire nuclear biology is much less complex. In insects at least, it seems as if the nuclear PIWI family protein is unique to Drosophila. What's interesting is that mammals also have a nuclear PIWI family protein but they don't have the Rhino homolog, so this might be a case of convergent evolution.

Rhiannon Macrae: Are the PIWI proteins themselves relatively well conserved?

Dr. Brennecke: Yes, you will find a PIWI clade protein basically in every animal, but there seems to be some variation as to whether they are nuclear or cytoplasmic. The data at the moment suggests that the cytoplasmic mechanism is the more ancient one. Dave Bartel has shown that Ping Pong, which is a cytoplasmic amplification system for piRNAs, is conserved down to sponges.

Rhiannon Macrae: Where do you see this field going in the future?

Dr. Brennecke: There's been some wonderful genetics done on transposon silencing, and elements of this pathway have not only been found in genetic screens by people working directly on transposons, but also by people like Ruth Lehmann and Gertrud Schüpbach working on germline development. When RNAi was discovered, all of these elements came together. We've started to define relatively robustly the features of this path and many of the general concepts have now been laid out. The challenge now is to bridge this to chromatin biology because at some point this pathway has to talk to the basic chromatin transcription machinery. A second huge challenge would be to understand at the mechanistic level how these proteins are working.

For example, piRNA biogenesis occurs to a large extent on the outer mitochondrial membrane. This gives you 
an idea about the complexity in cell biological terms between proteins that recruit the RNA to the mitochondrial surface, and the nucleus, and the RNA helicases feeding in the RNA. The challenge will be to combine genomics with computational biology, plus biochemistry and classic chromatin biology.

Rhiannon Macrae: Are you interested in branching from Drosophila to see if you can find piRNAs or similar pathways in other, perhaps nonmodel, organisms?

Dr. Brennecke: It's tempting, especially because of the abilities we have now with sequencing and short-read and long-read genome assemblies, and genome editing that can work in a wide range of species, but so far my lab has focused exclusively on Drosophila. I'm a passionate Drosophila geneticist and I just find that system so powerful. I don't impose myself on what people in the lab should do, but I think it requires the right people. If somebody comes in and has a very strong interest in setting up such research, I think it's the right time to do it.

Rhiannon Macrae: We are celebrating 150 years of Gregor Mendel's laws. Do you remember when you learned about Mendel's laws of inheritance or when you saw the Punnett square for the first time?

Dr. Brennecke: I probably learned it at school, but my first memory goes back to university where a friend of mine gave a seminar on Mendel's laws. Rather than talking about peas, he used two other traits that apparently are inherited in a Mendelian fashion; one was tonguerolling. The other was even more exotic. Apparently, when you eat asparagus, your pee smells, but there are people where it doesn't, so that's apparently a Mendelian trait as well. It was a memorable way to learn the subject. 


\section{$\$_{\text {CSH }}^{\infty}$ Cold Spring Harbor Symposia SYMPOSIA On Quantitative Biology}

\section{A Conversation with Julius Brennecke}

Cold Spring Harb Symp Quant Biol 2015 80: 302-304

Access the most recent version at doi:10.1101/sqb.2015.80.029967

\section{License}

Email Alerting Receive free email alerts when new articles cite this article - sign up in Service the box at the top right corner of the article or click here. 